\title{
Effects of the Short Foot Exercise With Neuromuscular Electrical Stimulation on Navicular Height in Flexible Flatfoot in Thailand: A Randomized Controlled Trial
}

\author{
Juntip Namsawang ${ }^{1}$, Wichai Eungpinichpong ${ }^{2}$, Ratana Vichiansiri ${ }^{3}$, Somchai Rattanathongkom ${ }^{1}$ \\ ${ }^{1}$ School of Physiotherapy, Faculty of Associated Medical Sciences, Khon Kaen University, Khon Kaen, Thailand; ${ }^{2}$ Research Center in Back, Neck and \\ Other Joint Pain and Human Performance (BNOJPH), Faculty of Associated Medical Sciences, Khon Kaen University, Khon Kaen, Thailand; \\ ${ }^{3}$ Department of Rehabilitation Medicine, Faculty of Medicine, Khon Kaen University, Khon Kaen, Thailand
}

Objectives: Flatfoot, or low medial longitudinal arch, contributes to back and lower extremity injuries and is caused by weak abductor hallucis (AbdH) muscles. The purpose of this study was to investigate the effects of short foot exercise (SFE) alone or with neuromuscular electrical stimulation (NMES) on navicular height, the cross-sectional area (CSA) of the AbdH muscle, and AbdH muscle activity in flexible flatfoot.

Methods: Thirty-six otherwise healthy people with flexible flatfoot were randomly assigned to a group that received SFE with placebo NMES treatment (the control group) or a group that received both SFE and NMES treatment (the experimental group). Each group received 4 weeks of treatment (SFE alone or SFE with NMES). Navicular height, the CSA of the AbdH muscle, and AbdH muscle activity were assessed before and after the intervention.

Results: No significant differences were found in navicular height or the CSA of the AbdH muscle between the control and experimental groups, while $\mathrm{AbdH}$ muscle activity showed a statistically significant difference between the groups (SFE $=73.9 \pm 11.0 \%$ of maximal voluntary isometric contraction [MVIC]; SFE with NMES $=81.4 \pm 8.3 \%$ of MVIC; $p<0.05$ ). Moreover, the CSA of the AbdH muscle showed a statistically significant increase after treatment in the SFE with NMES group (pre-treatment $=218.6 \pm 53.2 \mathrm{~mm}^{2}$; post-treatment $\left.=256.9 \pm 70.5 \mathrm{~mm}^{2} ; p<0.05\right)$.

Conclusions: SFE with NMES was more effective than SFE alone in increasing AbdH muscle activity. Therefore, SFE with NMES should be recommended to correct or prevent abnormalities in people with flexible flatfoot by a physiotherapist or medical care team.

Key words: Exercise, Electrical stimulation, Flatfoot, Randomized controlled trial, Thailand

Received: April 7, 2019 Accepted: June 28, 2019

Corresponding author: Somchai Rattanathongkom, PhD School of Physiotherapy, Faculty of Associated Medical Sciences, Khon Kaen University, 16 Mittraphap Road, Khon Kaen 40002, Thailand E-mail: somch_ra@kku.ac.th

This is an Open Access article distributed under the terms of the Creative Commons Attribution Non-Commercial License (http://creativecommons.org/licenses/by$\mathrm{nc} / 4.0 /$ ) which permits unrestricted non-commercial use, distribution, and reproduction in any medium, provided the original work is properly cited.

\section{INTRODUCTION}

Flatfoot, a common musculoskeletal disorder in adulthood, manifests as low or absent height of the medial longitudinal arch (MLA) while bearing weight, typically combined with forefoot abduction and rear foot eversion [1]. The prevalence of flexible flatfoot has been reported to be $20-30 \%$ [2]. In flexible flatfoot, changes of the foot shape result in overuse injuries of the back and lower extremity regions, as manifested by 
back pain [3], patellofemoral pain syndrome, and plantar fasciitis [4]. If left untreated, the foot deformity tends to progress and worsen over time, and eventually results in significant disability [5]. Structures including the tarsal and metatarsal bones, ligaments, plantar fascia, and the intrinsic and extrinsic foot muscles play an important role in supporting the MLA. Their main function is to stabilize the foot while bearing weight, standing, or walking. The extrinsic foot muscles are the prime movers of the foot [6], whereas the intrinsic foot muscles, such as the abductor hallucis $(\mathrm{AbdH})$, flexor digitorum brevis, and quadratus plantae, stabilize the MLA and control arch deformation [6-8].

The AbdH muscle is located within the first layer of the plantar surface of the foot. It originates on the posteromedial calcaneal tuberosity and inserts into the base of the proximal phalanx of the great toe [9]. It lies in the longitudinal axis of the foot and is perpendicular to the oblique axis of the transverse tarsal joint [8]. Contraction of this muscle increases flexion and supination of the first metatarsal, inversion of the calcaneus, and external rotation of the tibia in conjunction with elevation of the MLA [10]. Fiolkowski et al. [11] and Headlee et al. [12] demonstrated that impairment of AbdH muscle activity can result in an increase in the navicular drop. Thus, strengthening of the $\mathrm{AbdH}$ muscle is very important for promoting greater calcaneus inversion and elevation of the MLA $[7,10]$.

The currently used methods to strengthen the $\mathrm{AbdH}$ muscle are the towel curl exercise, short foot exercise (SFE), and neuromuscular electrical stimulation (NMES) [13]; however, SFE and NMES can only affect the plantar intrinsic foot muscles $[14,15]$. It was hypothesized that SFE and NMES training could increase $\mathrm{AbdH}$ muscle strength in people with flexible flatfoot and that SFE and NMES training could increase MLA height.

SFE is the most widely accepted method of strength training of the plantar intrinsic foot muscles. The exercise is performed by pulling the head of the metatarsal bones toward the calcaneus, without flexing the toes, and lifting the forefoot and heel from the ground [13]. Considerable evidence indicates that SFE could decrease the navicular drop [4], increase the activity of the plantar intrinsic foot muscles [14,16], and prevent the collapse of the MLA [4].

Additionally, evidence supports the widespread use of NMES as a safe method of stimulation in healthy adults, with or without muscle weakness. It was previously believed that electrical stimulation of the $\mathrm{AbdH}$ muscle could reduce the navicular drop after training [17] and could immediately induce foot inversion, which persisted for 2 months after the intervention [18]. However, other studies have found that the combination of NMES and exercise may increase muscle strength more than exercise alone [19-21]. As for the frequency of training, several studies have used 3-5 weeks of strength gains [17,22-24]. Fourchet et al. [17] found that 3 weeks of electrical stimulation of the intrinsic foot muscles significantly decreased the navicular drop. Canning and Grenier [22] and Parker et al. [23] showed that NMES was most effective in increasing muscle strength of the vastus medialis muscle and quadriceps femoris muscle, respectively, in healthy participants after 4 weeks.

No previous study has evaluated the short-term effects of SFE and NMES on the AbdH muscle in people with flexible flatfoot. Therefore, the purpose of this study was to investigate the effects of SFE alone or with NMES on navicular height, crosssectional area (CSA) of the AbdH muscle, and AbdH muscle activity in people with flexible flatfoot.

\section{METHODS}

\section{Design, Participants, and Randomization}

The study was a randomized controlled trial (RCT) with concealed allocation. The RCT was conducted from July 1, 2018 to October 31, 2018, at the physical therapy laboratory of in Burapha University, Thailand.

Among 60 volunteers who were originally recruited from Muang District, Chonburi Province, 24 were excluded based on the inclusion and exclusion criteria before commencement of the study, as shown in Figure 1. Thus, 36 participants with flexible flatfoot who met the inclusion criteria were randomly assigned to the experimental group (SFE with NMES) or the control group (SFE with placebo NMES). The demographic and clinical characteristics of the participants are shown in Table 1. The sample size was calculated based on previous studies [17] on electrical stimulation; in normal participants, we postulated that the average navicular drop post-intervention in the experimental and control groups would be $5.8 \pm 2.1 \mathrm{~mm}$ and $9.8 \pm$ $3.7 \mathrm{~mm}$, respectively. A statistical significance level of 0.05 and power of $90 \%$ were used in the sample size calculation, which yielded 18 participants in each group.

The inclusion criteria required participants to have been diagnosed (by an orthopedist) with flexible flatfoot. The study excluded all participants who had a history of foot and ankle 


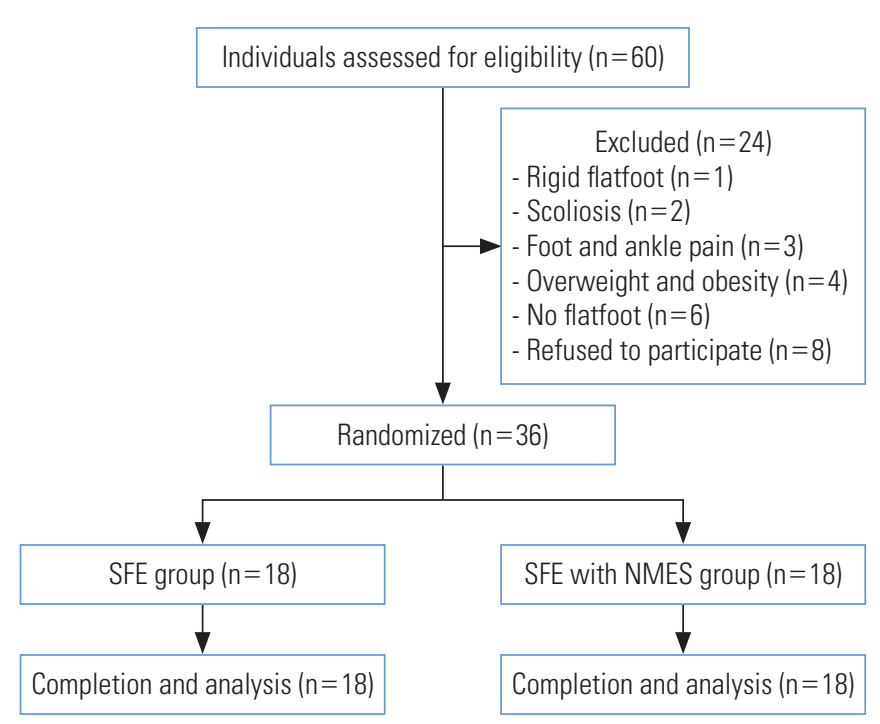

Figure 1. Flow chart of the participants. SFE, short foot exercise; NMES, neuromuscular electrical stimulation.

pain, genu valgum, femoral anteversion, posterior tibialis tendon dysfunction, leg length discrepancy, short gastrocnemius muscle, diabetes mellitus, rheumatoid arthritis, hypermobile joint, menopause, use of a cardiac pacemaker, broken or irritated skin at the electrode site, or a neurological disorder within the past 6 months.

After the baseline assessment was performed, the participants were randomly assigned to either the experimental or control group. The randomization schedule was prepared by a physiotherapist (researcher 1) using a computer-generated randomization sequence that randomly allocated participants using block sizes of 4 . A numeric block randomization sequence was placed in sealed opaque envelopes. The assessment staff was unaware of the design of the study and group allocation. The participants were explicitly informed and reminded not to discuss their randomization assignment with the assessment staff. Another physiotherapist (researcher 2) allocated the participants to groups and assigned the interventions.

\section{Interventions}

This experiment took a total of 14 days. On the first day (day 0 ), baseline data were measured by a third physiotherapist (researcher 3) before beginning the study the next day. Thereafter, the study was conducted 3 consecutive days (day 1, 2, and 3) per week, for 4 weeks. Both techniques (SFE with NMES) were applied by researcher 2 for 45 minutes during the 3 consecutive days. Post-treatment measurements were made after completing the last treatment on each day. Researcher 3 ,
Table 1. Baseline demographic and clinical characteristics of the participants

\begin{tabular}{lrcc}
\hline Variable & $\begin{array}{c}\text { SFE } \\
(\mathbf{n}=\mathbf{1 8})\end{array}$ & $\begin{array}{c}\text { SFE with } \\
\text { NMES (n=18) }\end{array}$ & $\boldsymbol{p}^{\text {-value }}{ }^{\mathbf{1}}$ \\
\hline Sex & & & 0.16 \\
$\quad$ Male & $4(22.2)$ & $8(44.4)$ & \\
$\quad$ Female & $14(77.8)$ & $10(55.6)$ & \\
Age (y) & $19.78 \pm 1.59$ & $20.17 \pm 1.20$ & 0.41 \\
Weight (kg) & $60.83 \pm 5.39$ & $58.17 \pm 5.99$ & 0.17 \\
Height (cm) & $168.78 \pm 6.15$ & $164.17 \pm 7.70$ & 0.06 \\
Body mass index (kg/m²) & $21.35 \pm 1.41$ & $21.57 \pm 1.50$ & 0.65 \\
Navicular height $(\mathrm{mm})$ & $32.76 \pm 4.17$ & $32.05 \pm 4.19$ & 0.54 \\
CSA of the AbdH muscle $\left(\mathrm{mm}^{2}\right)$ & $225.97 \pm 64.10$ & $218.66 \pm 53.23$ & 0.69 \\
AbdH muscle activity $(\% \mathrm{MVIC})$ & $65.29 \pm 12.89$ & $65.33 \pm 11.28$ & 0.99 \\
\hline
\end{tabular}

Values are presented as number (\%) or mean \pm standard deviation.

SFE, short foot exercise; NMES, neuromuscular electrical stimulation; CSA cross-sectional area; $\mathrm{AbdH}$, abductor hallucis; MVIC, maximal voluntary isometric contraction.

${ }^{1}$ Student $t$-test or chi-square test.

who remained blinded to the participants' group allocation, measured navicular height, the CSA of the AbdH muscle, and AbdH muscle activity.

The experimental group received SFE with NMES administered by researcher 2. Initially, participants performed SFE by placing both feet on the ground in the sitting position. They attempted to pull the head of the metatarsal bones toward the calcaneus without flexing their toes or lifting their forefoot and heel from the ground, and did so for 30 repetitions per day. Participants held each repetition for 5 seconds. Then, participants received NMES treatment on the AbdH muscle. Electrodes with a diameter of $38 \mathrm{~mm}$ were placed on the skin over the belly of the $\mathrm{AbdH}$ muscle using the bipolar technique. The active electrode was placed over the motor point of the $\mathrm{AbdH}$ muscle, while the dispersive electrode was placed behind the head of the first metatarsal bone $[18,25]$. The NMES electrodes were set to a high-voltage pulsed current, with a frequency of $85 \mathrm{~Hz}$, a frequency modulation of $90 \%$, a contraction time of 5 seconds, a rest time of 12 seconds, a ramp-up time of $0.3 \mathrm{sec}-$ onds, and a ramp-down time of 0.7 seconds; each session lasted for a total of 30 minutes. The intensity of NMES treatment was adjusted to the maximum tolerance of each participant while standing on both feet without pain or discomfort. The same researcher administered SFE and placebo NMES to the participants allocated to the control group in the same way as the experimental group, except that the electrodes provided no stimulation. Thus, the intensity of the placebo NMES was set at $0 \mathrm{~mA}$ while the participants stood on both feet. 


\section{Outcome Measurement}

The primary outcome of the treatment methods applied in this study was navicular height, which was analyzed by radiography (using lateral views) on day 0 and on each subsequent day after completing the last treatment session. In the navicular height measurements, participants stood on the platform with equal weight on both legs, with knees extended and feet relaxed, while radiological technicians placed the radiographic film between their feet. Navicular height is defined as the distance between the floor and the inferior border of the navicular bone [26]. All films were read by a radiologist.

The secondary outcomes included (1) CSA of the AbdH muscle and (2) AbdH muscle activity. These variables were measured pre- and post-intervention. A diagnostic ultrasound machine (M5 series; Shenzhen Mindray Bio-Medical Electronics Co., Shenzhen, China) with a 38-mm aperture and 7.5-MHz linear array probe (7L4s, Shenzhen Mindray Bio-Medical Electronics $\mathrm{Co}_{0}$ ) was used to analyze the CSA of the AbdH muscle. Participants lay down in the supine position, while their knees were supported by a pillow at approximately $15^{\circ}$ with their ankle in a neutral position [27]. A probe with ample gel was placed perpendicular to the $\mathrm{AbdH}$ muscle at $1 \mathrm{~cm}$ behind the navicular tuberosity $[28,29]$. The researcher optimized the procedure to obtain a clear image of the $\mathrm{AbdH}$ muscle. If the screen showed a clear picture, the image was captured. The probe was placed with minimal pressure, as slight pressure on the probe can lead to significant changes in muscle size. This measurement was performed 3 times. Investigators underwent 3 months of training in performing ultrasound scans of the AbdH muscle before performing data collection. Lastly, image processing was performed and the CSA was measured by tracing the muscle border $[27,29]$ of the AbdH muscle, using ImageJ version 1.51 (National Institutes of Health, Bethesda, MD, USA).

Additionally, wireless surface electromyography (EMG; TeleMyo DTS, Noraxon Inc., Scottsdale, AZ, USA) was used to measure the $A b d H$ muscle activity. The sample rate was set at 1000 $\mathrm{Hz}$, while the digital band-pass filter was set between $10 \mathrm{~Hz}$ to $500 \mathrm{~Hz}$. Root mean square values were calculated using a moving 50-ms window. The participants sat on a chair with hip and knee flexion at $90^{\circ}$ and ankles in a neutral position [12]. Then, the researcher cleaned the skin area to which the electrodes would be applied using a swab and alcohol before placing the surface electrodes on the $\mathrm{AbdH}$ muscle belly parallel to the muscle fibers over the motor point. These locations were determined according to the Surface Electromyography for the Non-Invasive Assessment of Muscles guidelines. The legs and feet receiving treatment were fixed with a Velcro strap to prevent trick movements, especially of the ankle joint. The participants flexed their hallux as hard as possible with 3 attempts for 5 seconds without lifting the heel and forefoot, performed distal phalanx flexion [11,12], and rested $60 \mathrm{sec}-$ onds between session [12]. The analysis of AbdH muscle activity used only the middle 3 seconds of the data, while the values from the first and last second were discarded. In order to normalize the EMG data, the maximal voluntary isometric contraction (MVIC) of the AbdH muscle was measured through manual muscle testing [30].

\section{Data Analysis}

Descriptive statistical analysis was used to describe participants' demographic and clinical data as mean \pm standard deviation. The Shapiro-Wilk test was used to explore the normality of all variables. The significance of differences in the baseline measurements between groups was tested by the Student $t$-test or the chi-square test. The mean changes in navicular height, the CSA of the AbdH muscle, and AbdH muscle activity between groups at baseline and at 4 weeks post-intervention were compared using the Student $t$-test. Statistical significance was set at $p$-value $<0.05$ for all tests.

\section{Ethics Statement}

This present study was approved by the Khon Kaen University Ethics Committee for Human Research (IRB00008614). All participants provided written informed consent.

\section{RESULTS}

In total, 36 participants with flexible flatfoot were recruited. No participants left the trial. Data from all 36 participants were analyzed. Compliance with the study intervention was excellent, including all 18 participants in the experimental group.

\section{Primary Outcome}

There was little increase in navicular height in either group (SFE $=0.04 \mathrm{~mm}$ and SFE with NMES $=0.09 \mathrm{~mm}$ ) and no statistically significant difference in navicular height between the experimental and control groups, as demonstrated in Figure 2A. 

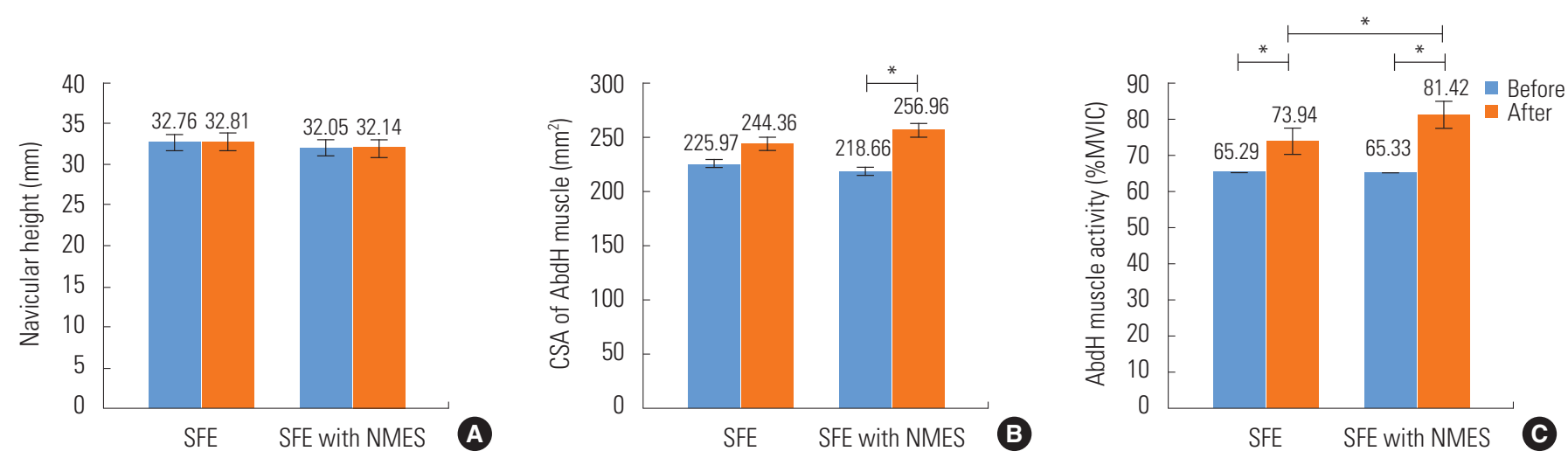

Figure 2. The change in (A) height of navicular tuberosity, (B) CSA of the AbdH muscle, and (C) AbdH muscle activity before and after treatment in the two groups. Values are presented as mean \pm standard deviation. SFE, short foot exercise; NMES, neuromuscular electrical stimulation; CSA, cross-sectional area; AbdH, abductor hallucis; MVIC, maximal voluntary isometric contraction. ${ }^{*} p<0.05$.

\section{Secondary Outcomes}

This study demonstrated an increase in the CSA of the AbdH muscle in both groups (SFE $=18.4 \mathrm{~mm}^{2}$ and SFE with NMES= $38.3 \mathrm{~mm}^{2}$ ). The average change in the CSA of the AbdH muscle in the group that received SFE with NMES was double that of the group that received SFE alone. However, this difference did not reach statistical significance (Figure 2B).

The findings did show a statistically significant increase in muscle strength in both groups (SFE $=73.9 \pm 11.0 \%$ of MVIC; SFE with NMES $=81.4 \pm 8.3 \%$ of MVIC; $p<0.05$ ). Moreover, AbdH muscle activity showed a statistically significant difference between the groups (mean difference $=7.48 \%$ of MVIC; $p<0.05$ ) (Figure 2C).

\section{DISCUSSION}

This is the first RCT to report the effectiveness of SFE with NMES in people with flexible flatfoot. The aim of this study was to investigate the effects of SFE with NMES on navicular height, the CSA of the AbdH muscle, and AbdH muscle activity in people with flexible flatfoot. Navicular height, as measured using radiography, showed a slight increase from baseline to post-treatment in both groups, with no significant difference between the groups. These findings are congruent with clinical measurements in previous reports showing no effect of intrinsic foot muscle training on navicular height.

Previous studies demonstrated that navicular height was not significantly affected by intrinsic foot muscle exercises in healthy participants [15], and electrical stimulation of the $\mathrm{AbdH}$ muscle in flatfoot participants yielded no change in na- vicular height [25]. Although no changes were found in navicular height, changes were found in the forefoot inversion angle and rear-foot adduction angle after training [25]. In addition, Okamura and colleagues [31] demonstrated that the timing of electrical stimulation had a slight effect on navicular height. Additionally, a study reported evidence that electrical stimulation of the AbdH muscle had a sufficient force to produce an angular displacement of the calcaneus and metatarsals and elevation of the MLA, thereby promoting MLA elevation [7].

To explain the lack of change in navicular height, the concept of a "closed kinetic chain" has been used. The navicular tuberosity and posterior calcaneus are defined as the reference points for clinical measurements of the subtalar joint. Changes in subtalar joint positions affect the navicular via the talus at the talonavicular joint. Therefore, the change in the height of the navicular tuberosity from the floor of the foot is related to changes in the calcaneus and subtalar joints [32]. A slight increase in AbdH muscle strength may not be sufficient to cause changes in the calcaneus and subtalar joints. Consequently, navicular height tended to change very little and did not achieve statistical significance.

However, we found that AbdH muscle activity significantly increased in the group that received SFE with NMES in comparison to the group that received SFE alone $(p<0.05)$. The CSA of the AbdH muscle increased significantly from baseline in the SFE with NMES group $(p<0.05)$. These results indicate that SFE with NMES was more effective for activating the AbdH muscle. Muscle activity plays an important role in muscle strength gains, which might be explained by the theory of 
adaptations within the nervous system [33], which predominate in the early phase of strength training, especially in electromyostimulation training lasting 4 weeks or less [34-36]. Our study is in agreement with previous studies that found a significant increase in muscle activity after 4 weeks of electromyostimulation training $[22,36]$. Exercise tended to increase muscle activity at 2 days after training [16]. Interestingly, our study found that the CSA of the AbdH muscle increased after 4 weeks of NMES training. Therefore, muscle hypertrophy can occur more rapidly than previously reported by Gondin et al. [34], who demonstrated that electromyostimulation increased the CSA of the knee extensors after 8 weeks of training. It is possible that the NMES program of our study was of sufficient intensity and duration to activate a greater proportion of fast twitch fibers than exercise alone. Type II muscle fibers are regarded as the key factor in achieving muscle hypertrophy [33].

The results of the present study clearly demonstrate that NMES may induce both neural and muscular adaptations in people with flexible flatfoot. Thus, NMES should have useful benefits in rehabilitation exercise programs aiming to increase intrinsic foot muscle strength. Flatfoot usually goes untreated. In addition to pain, it may cause significant long-term problems. For example, flatfoot may further aggravate knee pain and disability in people with knee osteoarthritis [37], and older adults had an increased risk of recurrent falls if their foot posture was planus [38]. Thus, our study helps to raise awareness about the clinical seriousness of flatfoot and possible treatment strategies.

Our study had some limitations. First, the results of this study cannot be generalized because the participants were young adults (average age, 20 years). Second, this study investigated only the short-term effects (4 weeks) of SFE and NMES in people with flexible flatfoot. Additionally, the changes in navicular height after the experiment were not clear. Thus, an experiment with a longer follow-up period is suggested. Moreover, changes in the forefoot and calcaneus angle may result from alterations in navicular height. Lastly, this study assessed the effect of strength training in asymptomatic cases of flexible flatfoot. However, the study was inconclusive regarding symptoms of flexible flatfoot. Thus we suggest further studies on the effects of treatment on people with symptomatic flexible flatfoot.

In conclusion, this present study demonstrated that SFE with NMES was more effective for increasing $\mathrm{AbdH}$ muscle strength than SFE alone. Although navicular height did not differ significantly between groups, we showed greater $\mathrm{AbdH}$ muscle activity and CSA of the AbdH muscle in the SFE with NMES group. These are important factors in the improved height of MLA. Therefore, we recommend SFE with NMES for use to correct or prevent abnormalities in people with flexible flatfoot by a physiotherapist or medical care team.

\section{CONFLICT OF INTEREST}

The authors have no conflicts of interest associated with the material presented in this paper.

\section{ACKNOWLEDGEMENTS}

This work was supported by Faculty of Associated Medical Sciences, Khon Kaen University.

\section{AUTHOR CONTRIBUTIONS}

Conceptualization: JN, WE, RV, SR. Data curation: JN, SR. Formal analysis: JN, SR. Funding acquisition: JN, SR. Methodology: JN, WE, RV, SR. Project administration: JN, SR. Visualization: JN, SR. Writing - original draft: JN, SR. Writing - review \& editing: $J N, W E, R V$, SR.

\section{ORCID}

Juntip Namsawang https://orcid.org/0000-0002-5365-197X

Wichai Eungpinichpong https://orcid.org/0000-0001-70141448

Ratana Vichiansiri https://orcid.org/0000-0002-7578-0481

Somchai Rattanathongkom https://orcid.org/0000-00021386-353X

\section{REFERENCES}

1. Mercier L. Practical orthopedics. 6th ed. Philadelphia: Mosby; 2008, p. 224.

2. Rachmawati MR, Tulaar AB, Immanuel S, Purba A, Mansyur $M$, Haryadi RD, et al. Correcting of pronated feet reduce skeletal muscle injury in young women with biomechanical abnormalities. Anat Cell Biol 2016;49(1):15-20.

3. Menz HB, Dufour AB, Riskowski JL, Hillstrom HJ, Hannan MT. Foot posture, foot function and low back pain: the Framingham Foot Study. Rheumatology (Oxford) 2013;52(12):2275- 
2282.

4. Mulligan EP, Cook PG. Effect of plantar intrinsic muscle training on medial longitudinal arch morphology and dynamic function. Man Ther 2013;18(5):425-430.

5. Henry JK, Shakked R, Ellis SJ. Adult-acquired flatfoot deformity. Foot Ankle Orthop 2019;4(1):1-17.

6. McKeon PO, Hertel J, Bramble D, Davis I. The foot core system: a new paradigm for understanding intrinsic foot muscle function. Br J Sports Med 2015;49(5):290.

7. Kelly LA, Cresswell AG, Racinais S, Whiteley R, Lichtwark G. Intrinsic foot muscles have the capacity to control deformation of the longitudinal arch. J R Soc Interface 2014;11(93):20131188.

8. Mann R, Inman Vt. Phasic activity of intrinsic muscles of the foot. J Bone Joint Surg 1964;46(3):469-481.

9. Agur AM, Dalley AF. Grant's atlas of anatomy. 14th ed. Philadelphia: Wolters Kluwer; 2017, p. 558.

10. Wong YS. Influence of the abductor hallucis muscle on the medial arch of the foot: a kinematic and anatomical cadaver study. Foot Ankle Int 2007;28(5):617-620.

11. Fiolkowski P, Brunt D, Bishop M, Woo R, Horodyski M. Intrinsic pedal musculature support of the medial longitudinal arch: an electromyography study. J foot Ankle Surg 2003;42(6):327333.

12. Headlee DL, Leonard JL, Hart JM, Ingersoll CD, Hertel J. Fatigue of the plantar intrinsic foot muscles increases navicular drop. J Electromyogr Kinesiol 2008;18(3):420-425.

13. Starkey C. Athletic training and sports medicine: an integrated approach. 5th ed. Burlington: Jones \& Bartlett Learning; 2013, p. 58.

14. Jung DY, Kim MH, Koh EK, Kwon OY, Cynn HS, Lee WH. A comparison in the muscle activity of the abductor hallucis and the medial longitudinal arch angle during toe curl and short foot exercises. Phys Ther Sport 2011;12(1):30-35.

15. Lynn SK, Padilla RA, Tsang KK. Differences in static- and dynamic-balance task performance after 4 weeks of intrinsicfoot-muscle training: the short-foot exercise versus the towelcurl exercise. J Sport Rehabil 2012;21(4):327-333.

16. Gooding TM, Feger MA, Hart JM, Hertel J. Intrinsic foot muscle activation during specific exercises: a T2 time magnetic resonance imaging study. J Athl Train 2016;51(8):644-650.

17. Fourchet F, Kilgallon M, Loepelt H, Millet GP. Plantar muscles electrostimulation and navicular drop. Sci Sports 2009;24(5): 262-264 (French).

18. Gaillet JC, Biraud JC, Bessou M, Bessou P. Modifications of baropodograms after transcutaneous electric stimulation of the abductor hallucis muscle in humans standing erect. Clin Biomech (Bristol, Avon) 2004;19(10):1066-1069.

19. Alon G, Taylor DJ. Electrically elicited minimal visible tetanic contraction and its effect on abdominal muscles strength and endurance. Eur J Phys Med Rehabil 1997;7(1):2-6.

20. Kim KM, Croy T, Hertel J, Saliba S. Effects of neuromuscular electrical stimulation after anterior cruciate ligament reconstruction on quadriceps strength, function, and patient-oriented outcomes: a systematic review. J Orthop Sports Phys Ther 2010;40(7):383-391.

21. Selkowitz DM. Improvement in isometric strength of the quadriceps femoris muscle after training with electrical stimulation. Phys Ther 1985;65(2):186-196.

22. Canning A, Grenier S. Does neuromuscular electrical stimulation improve muscular strength gains of the vastus medialis muscle. Int J Phys Med Rehabil 2014;2:207.

23. Parker MG, Bennett MJ, Hieb MA, Hollar AC, Roe AA. Strength response in human femoris muscle during 2 neuromuscular electrical stimulation programs. J Orthop Sports Phys Ther 2003;33(12):719-726.

24. Fourchet F, Gojanovic B. Foot core strengthening: relevance in injury prevention and rehabilitation for runners. Swiss Sports Exerc Med 2016;64(1):26-30.

25. Okamura K, Kanai S, Fukuda K, Tanaka S, Ono T, Oki S. The effect of additional activation of the plantar intrinsic foot muscles on foot kinematics in flat-footed subjects. Foot (Edinb) 2019;38:19-23.

26. Menz HB, Munteanu SE. Validity of 3 clinical techniques for the measurement of static foot posture in older people. J Orthop Sports Phys Ther 2005;35(8):479-486.

27. Stewart S, Ellis $R$, Heath M, Rome K. Ultrasonic evaluation of the abductor hallucis muscle in hallux valgus: a cross-sectional observational study. BMC Musculoskelet Disord 2013;14:45.

28. Cameron AF, Rome K, Hing WA. Ultrasound evaluation of the abductor hallucis muscle: reliability study. J Foot Ankle Res 2008;1(1):12.

29. Lobo CC, Marín AG, Sanz DR, López DL, López PP, Morales CR, et al. Ultrasound evaluation of intrinsic plantar muscles and fascia in hallux valgus: a case-control study. Medicine (Baltimore) 2016;95(45):e5243.

30. Kendall FP, Crosby RW, Krause CC, McCreary EK. Muscles: testing and function. 3rd ed. Baltimore: Williams \& Wilkins; 1983, p. 131.

31. Okamura K, Kanai S, Hasegawa M, Otsuka A, Oki S. The effect of additional activation of the plantar intrinsic foot muscles 
on foot dynamics during gait. Foot (Edinb) 2018;34:1-5.

32. Sell KE, Verity TM, Worrell TW, Pease BJ, Wigglesworth J. Two measurement techniques for assessing subtalar joint position: a reliability study. J Orthop Sports Phys Ther 1994;19(3):162167.

33. Neumann DA. Kinesiology of the musculoskeletal system: foundations for physical rehabilitation. 2nd ed. St. Louis: Mosby/Elsevier; 2010, p. 69-70.

34. Gondin J, Guette M, Ballay Y, Martin A. Electromyostimulation training effects on neural drive and muscle architecture. Med Sci Sports Exerc 2005;37(8):1291-1299.

35. Singer KP. The influence of unilateral electrical muscle stimu- lation on motor unit activity patterns in atrophic human quadriceps. Aust J Physiother 1986;32(1):31-37.

36. Maffiuletti NA, Pensini M, Martin A. Activation of human plantar flexor muscles increases after electromyostimulation training. J Appl Physiol (1985) 2002;92(4):1383-1392.

37. lijima $\mathrm{H}$, Ohi $\mathrm{H}$, Isho $T$, Aoyama $T$, Fukutani $\mathrm{N}$, Kaneda $\mathrm{E}$, et al. Association of bilateral flat feet with knee pain and disability in patients with knee osteoarthritis: a cross-sectional study. J Orthop Res 2017;35(11):2490-2498.

38. Awale A, Hagedorn TJ, Dufour AB, Menz HB, Casey VA, Hannan MT. Foot function, foot pain, and falls in older adults: the Framingham foot study. Gerontology 2017;63(4):318-324. 